This item was submitted to Loughborough's Research Repository by the author.

Items in Figshare are protected by copyright, with all rights reserved, unless otherwise indicated.

\title{
Robust contact force controller for slip prevention in a robotic gripper
}

PLEASE CITE THE PUBLISHED VERSION

http://dx.doi.org/10.1243/09596518JSCE854

PUBLISHER

(C) IMechE

VERSION

VoR (Version of Record)

LICENCE

CC BY-NC-ND 4.0

REPOSITORY RECORD

O'Toole, Michael D., Kaddour Bouazza-Marouf, David Kerr, and Michael Vloeberghs. 2019. "Robust Contact Force Controller for Slip Prevention in a Robotic Gripper". figshare. https://hdl.handle.net/2134/12516. 
This item was submitted to Loughborough's Institutional Repository (https://dspace.lboro.ac.uk/) by the author and is made available under the following Creative Commons Licence conditions.

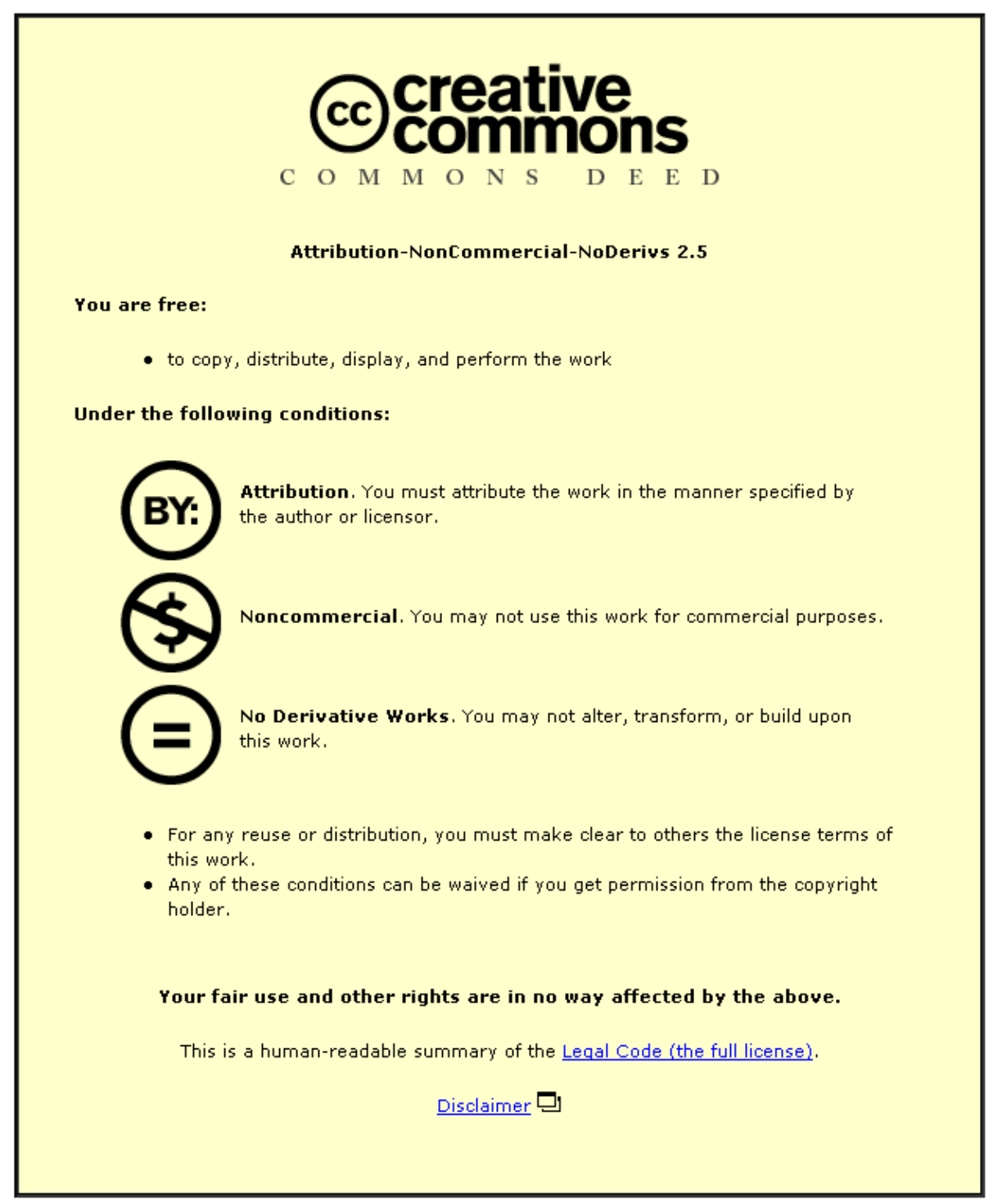

For the full text of this licence, please go to: http://creativecommons.org/licenses/by-nc-nd/2.5/ 


\title{
Robust contact force controller for slip prevention in a robotic gripper
}

\author{
M O’Toole $^{1 *}$, K Bouazza-Marouf ${ }^{1}$, D Kerr $^{1}$, and M Vloeberghs ${ }^{2}$ \\ ${ }^{1}$ Wolfson School, Loughborough University, Loughborough, UK \\ ${ }^{2}$ School of Human Development, University Hospital, Nottingham, UK
}

The manuscript was received on 16 July 2009 and was accepted after revision for publication on 11 January 2010.

DOI: 10.1243/09596518JSCE854

\begin{abstract}
Grasping a soft or fragile object requires the use of minimum contact force to prevent damage or deformation. Without precise knowledge of object parameters, real-time feedback control must be used with a suitable slip sensor to regulate the contact force and prevent slip. Furthermore, the controller must be designed to have good performance characteristics to rapidly modulate the fingertip contact force in response to a slip event. In this paper, a fuzzy sliding mode controller combined with a disturbance observer is proposed for contact force control and slip prevention. The controller is based on a system model that is suitable for a wide class of robotic gripper configurations. The robustness of the controller is evaluated through both simulation and experiment. The control scheme was found to be effective and robust to parameter uncertainty. When tested on a real system, however, chattering phenomena, well known to sliding mode research, was induced by the unmodelled suboptimal components of the system (filtering, backlash, and time delays), and the controller performance was reduced.
\end{abstract}

Keywords: sliding mode control, fuzzy logic, soft-grasping

\section{INTRODUCTION}

The soft-grasping problem requires a gripper to exert minimum contact force to maintain a static grasp with an object in optimal time and with minimal overshoot. If the precise characteristics of the gripper and object are known, e.g. weight, friction coefficient, etc., the optimal contact force can be calculated. This is feasible in applications where the object parameters remain consistent. However, this is impractical in most circumstances. The problem of slip prevention in robotic grippers is twofold:

(a) a suitable robust sensor to provide feedback on object slip;

(b) a real-time feedback controller for optimum contact force for slip prevention.

*Corresponding author: Wolfson School, Loughborough University, Loughborough, Leicestershire LE11 3TU, UK.

email: M.D.OToole@lboro.ac.uk
Detecting the slippage of an object requires a suitable sensor to convert slip into an output signal. Sensors tend to be structured to detect object motion relative to the sensor (slip rate), vibration caused by stick slip, or vibration caused by partial slip (incipient slip). Partial slip is a particularly useful measure as it can be detected when the velocity of the object relative to the gripper is zero $[\mathbf{1 - 5}]$. However, these sensors tend to be quite complex and require an uneven pressure distribution across the sensor, which may be suboptimal for gripping irregular-shaped objects. Object motion [6, 7] and stick slip vibration [8-11] require relatively simple and inexpensive sensors. The simplest form of these sensors is based on the rolling contact principle [6, 7] where slip induces some measurable rotation in the sensor. These sensors use uncomplicated designs and can be assembled from off-the-shelf components. The stick slip vibration sensor can be used for both detecting the occurrence of slip and also, through the design of a suitable observer or algorithm, determine the object slip rate. A controller with slip rate and/or slip displacement as 
state variables is compatible with a wide range of slip sensors. Therefore, the development of a contact force controller need not be sensor specific. However, vibration is also dependent on surface conditions and the robustness of the observer or algorithm to variation in this parameter must be considered.

In general, three different control strategies have been applied for the real-time soft-grasping problem.

1. Direct and linear-feedback control.

2. Force-ratio control.

3. Fuzzy-logic-based control.

Direct and linear feedback controllers use the slip signal to directly control grasp force. The most basic example of this type of control is simple on-off control, where the detection of slip triggers an event such as 'close gripper command' [11, 12]. Linear feedback controllers have also been used and have shown an improvement over simple on-off approaches. Engeberg and Meek [13] used proportional and proportional-derivative (PD) controllers to regulate shear force magnitude detected by strain gauges in a robotic hand. The scheme was further refined by a logic-based adaptive mechanism that increases the controller gain when a slip event is detected. Kyberd et al. [8] and Kyberd and Chappell [14] used a form of integral controller to control grasp force in a prosthetic hand. This method is based on the slip rate, inferred from the rate of pulses produced by a microphone-based slip sensor. The incremental count of the pulses is then used to define a grasp force demand signal. The difficulty with using linear-feedback controllers in the control of slip is that their gains are largely derived arbitrarily, and their robustness to parameter variations is unknown. Consequently, the controller gains would have to be retuned when the object or environment differed substantially from laboratory conditions.

Force ratio control uses knowledge of shear force and the controlled contact force (normal force) to maintain a constant ratio between the two equivalent to the coefficient of friction. The ratio is specific to the object-gripper interface, and some means of approximation is required. A partial slip sensor is particularly useful for this as it is able to detect the onset of slip, without movement of the grasped object. For example, Maekawa et al. [15] used this approach in a two-fingered gripper to augment a demand signal received from an openloop high-level grasp approximation scheme. Simi- larly, Koda and Maeno [5] used the same approach to modify a demand signal imposed by a human operator in a master-slave system. Force ratio control, however, requires a sensor that is able to measure both contact force, shear force, and some characteristic of slip. The incorporation of additional sensory requirements in the limited fingertip envelope is technically demanding.

Fuzzy-logic controllers are a popular example of a model-free approach to uncertain or non-linear control problems. They are appealing in this application as they replace a model with a heuristic rule set, circumventing the need for knowledge of the object properties such as mass and friction. Shang et al. [9] designed a fuzzy-logic controller (FLC) for use with outputs of a photoelastic slip sensor in a robotic gripper. In Dubey et al. [10], a gripper with an FLC and photoelastic slip sensor was used to find the optimal grasp force. The FLC was combined antagonistically with a relaxation function - which incrementally decreased the demand signal - to optimize the contact force around the point of slip. Glossas and Aspragathos [16] designed an FLC using a rule set derived empirically from human responses. The FLC design was shown through simulation to be superior to an empirically tuned proportional-integral-derivative controller.

Where a model cannot be easily developed, an FLC may be employed as an alternative control solution. The ability to use heuristic rules is appealing in robotic grasping, as it allows the designer to utilize results from neurophysiology, producing controllers based on 'as a human thinks' designs [16]. However, FLCs are not optimum solutions, but rather 'good enough' alternatives [17]. The membership functions must be defined arbitrarily, yielding suboptimal controller outputs, and the lack of a model makes it difficult to assess their robustness to differing circumstances. This problem was partially solved by Domínguez-López et al. [6] who proposed a neuro-fuzzy logic controller capable of retuning online. However, this scheme still required the use of training data.

In this paper, a sliding mode controller with a fuzzy sliding surface - referred to herein as a fuzzy sliding mode controller - suitable for a wide class of gripper configurations is designed for contact force control and slip prevention. Sliding mode control is an effective technique for rapid dynamic response of control systems with bounded parameter uncertainty and external disturbances [18]. It has proved useful in the analogous task of regulating a constant smooth slip rate despite the existence of stick slip 
[19]. By using a fuzzy sliding surface and disturbance observer, performance can be enhanced in the face of unmatched uncertainty, i.e. uncertainty outside of the input channel. In the remainder of this paper, the control strategy is designed based on a model of a generic gripper. The controller properties and performance are explored in section 3 through simulation of a robotic gripper which includes suboptimal but realistic constraints such as time delays and backlash. Issues relating to the practical implementation of the scheme are also discussed in this section, using results from an experimental test rig.

\section{ROBOTIC GRIPPER CONTROLLER SYNTHESIS}

The control strategy for the gripper is shown in Fig. 1. It consists of a disturbance observer which estimates the optimum gripper position to grasp the object without slip. The output of the observer forms the reference demand signal for a fuzzy sliding mode controller (FSMC) that uses slip rate $(\dot{x}(t))$, slip $(x(t))$, gripper position $(\theta(t))$, and gripper velocity $(\dot{\theta}(t))$ as state-feedback variables. The sliding mode control law drives the system state trajectories onto a predefined sliding surface using a relay. When the state trajectories are in contact with the sliding surface a so-called 'sliding motion' along the sliding surface occurs. The sliding surface is designed so that, when in sliding motion, the system exhibits ideal dynamic characteristics and is invariant to matched uncertainty. In order to improve robustness to unmatched uncertainty, the sliding plane is partially estimated using a Takagi-Sugeno fuzzylogic system (FLS). The FLS uses a heuristic rule set to evaluate fuzzified slip $(x(t))$ and slip rate $(\dot{x}(t))$ variables and derives an approximate sliding plane vector. As the slip and slip rate increase, the FLC makes the sliding plane more sensitive to occurrences of slipping. The gripper fingertip motion when the finger is in contact with the object is modelled by

$$
J_{\mathrm{e}} \ddot{\theta}(t)=\tau(t)-D_{\mathrm{e}} \dot{\theta}(t)-n K \delta l(t)+f_{\mathrm{m}}(\tau, t)
$$

where $K$ is the combined object and fingertip stiffness, $D_{\mathrm{e}}$ is the effective damping of the mechanism, $J_{\mathrm{e}}$ the effective inertia of the mechanism, $n$ is a load transmission constant, and $\tau(t)$ is the motor torque. $\delta l(t)$ is the linear deflection of the compliant elements of the gripper and object. As it is assumed to be small, it can be approximated from the gripperfinger angle as

$$
\delta l(t)=L\left(\theta(t)-\theta_{0}\right)
$$

where $\theta(t)$ is the gripper-finger angle from fully open position (i.e. $\theta(t)=0$ when the gripper is fully open), $\theta_{0}$ is the gripper-finger angle at initial contact with

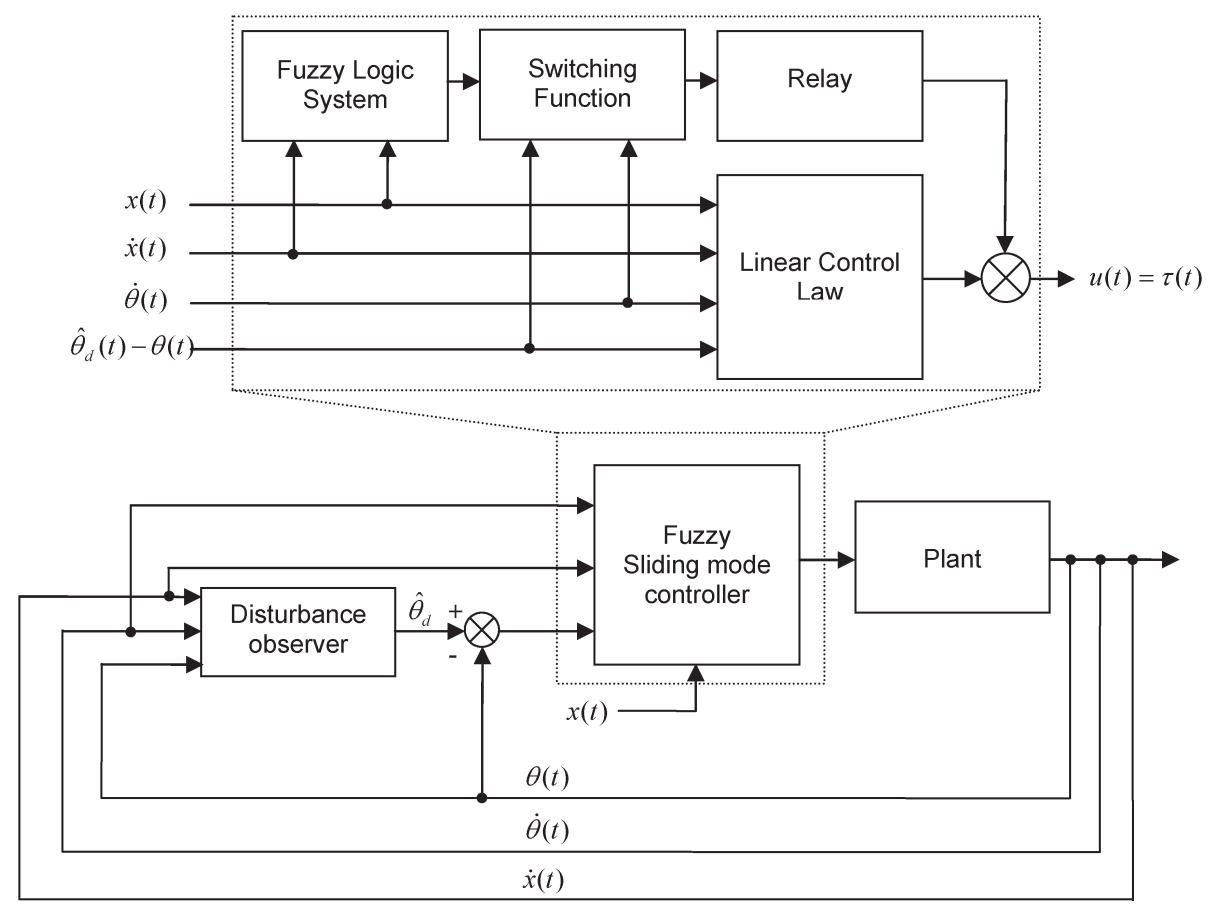

Fig. 1 Control system 
the object, and $L$ is the length from the gripper axis to the point of contact. It can be easily shown that equation (1) applies to a broad range of gripper types. The additional term $f_{\mathrm{m}}(\tau, t)$ represents system uncertainties, such as plant-model parameter mismatch, and unmodelled dynamics or disturbances.

As discussed in section 1, slip and slip rate can be directly detected or inferred by a large number of sensors. Thus, the choice of state-feedback variables and the system equation for the gripper can be applied to a broad set of gripper-sensor configurations. The free-body diagram for the grasped object is shown in Fig. 2. For convenience, the coefficient of friction $\mu(\dot{x})$ is linearized about the static friction coefficient so that $\mu(\dot{x})=\mu_{\mathrm{s}}+\Delta \mu(\dot{x})$. Then, using this assumption, object slip rate $\dot{x}(t)$ is governed by

$$
\ddot{x}(t)=-\frac{D_{\mathrm{s}}}{M_{\mathrm{obj}}} \dot{x}(t)-\frac{\mu_{\mathrm{s}}}{M_{\mathrm{obj}}} K \delta l(t)+\Psi(\dot{x}(t), \theta(t), t)
$$

where $M_{\mathrm{obj}}$ is the grasped object mass, $D_{\mathrm{s}}$ is the viscous coefficient at the site of contact between the object and the gripper, $\delta l$ is the linear deflection of the compliant elements of the gripper and object and is approximated by equation (2). $\Psi(\dot{x}(t)$, $\theta(t), t)$ is given by

$$
\begin{aligned}
\Psi(\dot{x}(t), \theta(t), t)= & \ddot{x}_{\mathrm{g}}(t)+\alpha g-\frac{1}{M_{\mathrm{obj}}} K \delta l(t) \Delta \mu(\dot{x}) \\
& +f_{\mathrm{s}}(\dot{x}(t), \theta(t), t)
\end{aligned}
$$

where $\ddot{x}_{\mathrm{g}}(t)$ is gripper acceleration, and $\alpha$ is a factor between zero and one. It is the cosine angle between the direction of the slip of the object relative to the vertical. $\Psi(\dot{x}(t), \theta(t), t)$ is an uncertainty term that includes the changes in the friction coefficient, gripper acceleration, direction of gravity parameter $(\alpha)$, and the function $f_{\mathrm{s}}(\dot{x}(t), \theta(t), t)$ represents model-plant parameter mismatch, or unmodelled dynamics and disturbances. In certain circumstances, gravitational force and acceleration of the object may be measured using sensors or estimated from a robot trajectory controller. However, this is not always practical and in order to apply the controller to a wide class of grippers, these variables will be considered unknown. For controller synthesis, it is assumed that both uncertainty terms $f_{\mathrm{m}}(\tau, t)$ and $\Psi(\dot{x}(t), y(t), t)$ are finite and subject to the conditions

$$
\begin{aligned}
& \left\|f_{\mathrm{m}}(\tau, t)\right\|<\tilde{f}(t) \\
& \|\Psi(\dot{x}(t), y(t), t)\|<\tilde{\Psi}(t)
\end{aligned}
$$

where $\tilde{f}(t)$ and $\tilde{\Psi}(t)$ are bounds on the uncertainty, and must be chosen according to the likely operating environment of the gripper.

\subsection{Disturbance observer}

A disturbance observer is used to find a suitable reference demand signal that can compensate for the unmatched system uncertainty. Using equation (3) the following algorithm is proposed to produce an estimate of the uncertainty term $\hat{\Psi}$

$$
\hat{\Psi}=\left[\ddot{x}(t)+\frac{D_{\mathrm{s}}}{M_{\mathrm{obj}}} \dot{x}(t)+\frac{\mu_{\mathrm{s}}}{M_{\mathrm{obj}}} K L\left(\theta(t)-\theta_{0}\right)\right]
$$

In the steady state, i.e. where no slip is occurring and the gripper is at rest in its optimum position, the uncertainty term in equation (4) can be written as

$$
\Psi(\dot{x}(t), \theta(t))=\frac{\mu_{\mathrm{s}}}{M_{\mathrm{obj}}} K L\left(\theta_{\mathrm{d}}-\theta_{0}\right)
$$

where $\theta_{\mathrm{d}}$ is the optimum gripper rest position and thus is the desired reference position. From this
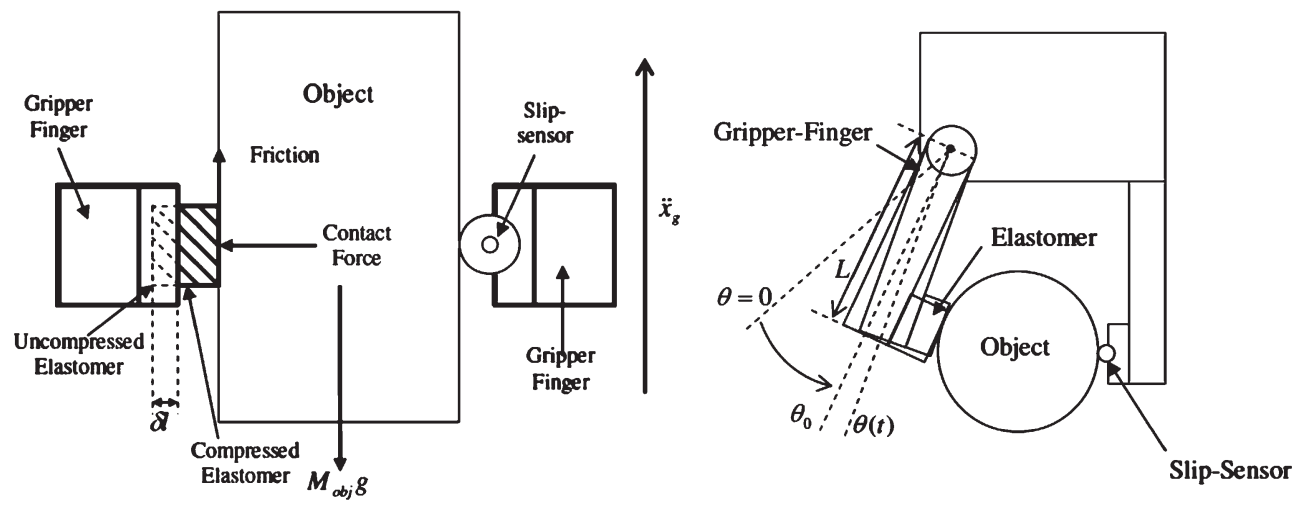

Fig. 2 Robotic gripper grasping an object 
result, the disturbance observer is reconfigured to produce an estimate of the optimum gripper position $\hat{\theta}_{\mathrm{d}}$ that cancels the unmatched uncertainty. Thus, the following disturbance observer is proposed

$$
\begin{aligned}
& \dot{z}=\mathbf{F} z+\mathbf{E} q \\
& q=\frac{M_{\mathrm{obj}}}{\mu_{\mathrm{s}} K L}\left[\ddot{x}(t)+\frac{D_{\mathrm{s}}}{M_{\mathrm{obj}}} \dot{x}(t)+\frac{\mu_{\mathrm{s}}}{M_{\mathrm{obj}}} K L\left(\theta(t)-\theta_{0}\right)\right] \\
& z=\hat{\theta}_{\mathrm{d}}-\theta_{0}
\end{aligned}
$$

where $\mathbf{F}, \mathbf{E}$ are system parameter matrices for a recursive low-pass filter that removes high-frequency measurement noise and disturbances and $z$ its internal variable. For the convergence of the observer the filter the parameters should be chosen such that $\mathbf{F}<0, \mathbf{E}>0$.

\subsection{Sliding mode controller}

The controller is designed according to the sliding mode controller methodology proposed in Edwards and Spurgeon [18]. Choosing partitioned state variable $\boldsymbol{x}=\left[\boldsymbol{x}_{1} \mid \boldsymbol{x}_{2}\right]^{\mathrm{T}}=\left[\begin{array}{ll}\dot{x}(t) & \theta(t)-\theta_{0} \mid \dot{\theta}(t)\end{array}\right]^{\mathrm{T}}$, input variable $u(t)=\tau(t)$, unmatched uncertainty vector

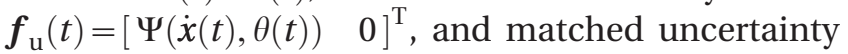
as $f_{\mathrm{m}}(t)$, equations (1) and (3) can be put in the form

$$
\begin{aligned}
& \dot{\boldsymbol{x}}_{1}=\mathbf{A}_{11} \boldsymbol{x}_{1}+\mathbf{A}_{12} \boldsymbol{x}_{2}+\boldsymbol{f}_{\mathrm{u}}(t) \\
& \dot{\boldsymbol{x}}_{2}=\mathbf{A}_{21} \boldsymbol{x}_{1}+\mathbf{A}_{22} \boldsymbol{x}_{2}+\mathbf{B}_{2} u(t)+\boldsymbol{f}_{\mathrm{m}}(t)
\end{aligned}
$$

$$
\begin{aligned}
& \mathbf{A}=\left[\begin{array}{l|l}
\mathbf{A}_{11} & \mathbf{A}_{12} \\
\hline \mathbf{A}_{21} & \mathbf{A}_{22}
\end{array}\right]=\left[\begin{array}{cc|c}
-\frac{D_{\mathrm{s}}}{M_{\mathrm{obj}}} & -\frac{\mu}{M_{\mathrm{obj}}} & 0 \\
0 & 0 & 1 \\
\hline 0 & -\frac{\eta K}{J} & -\frac{D}{J}
\end{array}\right] \\
& \mathbf{B}=\left[\frac{0}{\mathbf{B}_{2}}\right]=\left[\begin{array}{l}
0 \\
\frac{0}{1} \\
\frac{0}{J}
\end{array}\right]
\end{aligned}
$$

Introducing an error-state vector $\boldsymbol{e}=\left[\boldsymbol{e}_{1} \mid \boldsymbol{e}_{2}\right]^{\mathrm{T}}$

$$
\begin{aligned}
& \boldsymbol{e}_{1}=\boldsymbol{x}_{1}-\left[\begin{array}{ll}
0 & \hat{\theta}_{\mathrm{d}}-\theta_{0}
\end{array}\right]^{\mathrm{T}}=\left[\begin{array}{ll}
\dot{x}(t) & \theta(t)-\hat{\theta}_{\mathrm{d}}
\end{array}\right]^{\mathrm{T}} \\
& \boldsymbol{e}_{2}=\dot{\theta}(t)
\end{aligned}
$$

Defining a switching function as $\sigma(t)=$
$\left[S_{11} S_{22}\right] \boldsymbol{e}_{1}+\boldsymbol{S}_{2} \boldsymbol{e}_{2}$, and a sliding manifold as $\sigma=0$. Using the switching function, the first derivative with respect to time of the switching function, and equation (9), it can be shown that

$$
\begin{aligned}
\dot{\boldsymbol{e}}_{1}= & \overline{\mathbf{A}}_{11} \boldsymbol{e}_{1}+\mathbf{A}_{12} \mathbf{S}_{2}^{-1} \sigma(t)+\boldsymbol{f}_{\mathrm{u}}(t) \\
\dot{\boldsymbol{\sigma}}(t)= & \mathbf{S}_{2} \overline{\mathbf{A}}_{21} \boldsymbol{e}_{1}+\mathbf{S}_{2} \overline{\mathbf{A}}_{22} \mathbf{S}_{2}^{-1} \boldsymbol{\sigma}(t)+\mathbf{S}_{2} \mathbf{B}_{2} u(t) \\
& +\mathbf{S}_{2} \boldsymbol{f}_{\mathrm{m}}(t)+\mathbf{S}_{1} \boldsymbol{f}_{\mathrm{u}}(t)
\end{aligned}
$$

where $\quad \overline{\mathbf{A}}_{11}=\mathbf{A}_{11}-\mathbf{A}_{12} \mathbf{S}_{2}^{-1} \mathbf{S}_{1}, \quad \overline{\mathbf{A}}_{21}=\mathbf{S}_{2}^{-1} \mathbf{S}_{1} \overline{\mathbf{A}}_{11}+\mathbf{A}_{21}-$ $\mathbf{A}_{22} \mathbf{S}_{2}^{-1} \mathbf{S}_{1}$, and $\overline{\mathbf{A}}_{22}=\mathbf{S}_{2}^{-1} \mathbf{S}_{1} \mathbf{A}_{12}+\mathbf{A}_{22}$. A sliding mode controller of the form $u(t)=u_{\mathrm{L}}(x(t), t)+u_{\mathrm{N}}(s(t), t)$ is introduced, where the linear component $u_{\mathrm{L}}$ and discontinuous component $u_{\mathrm{N}}$ are

$$
\begin{aligned}
& u_{\mathrm{L}}(x(t), t)=-(\mathbf{S B})^{-1}(\mathbf{S A}-\Phi \mathbf{S}) \boldsymbol{e}(t) \\
& u_{\mathrm{N}}(\sigma(t), t)=-\rho(\mathbf{S B})^{-1} \operatorname{sgn}(\sigma(t))
\end{aligned}
$$

where $\rho$ is the discontinuity gain and $\Phi$ is the linear rate of decay onto the sliding surface. Choosing a Lyapunov candidate as $V=\sigma(t)^{2} / 2$. By substitution of equation (10) and the sliding mode controller in (11), the derivative of the Lyapunov candidate becomes

$$
\begin{aligned}
& \dot{V}=\sigma(t) \dot{\sigma}(t) \\
& \dot{V}=\sigma(t)\left(\Phi \sigma(t)-\rho \operatorname{sgn}(\sigma(t))+\mathbf{S}_{1} \boldsymbol{f}_{\mathrm{u}}(t)+\mathbf{S}_{2} \boldsymbol{f}_{\mathrm{m}}(t)\right)
\end{aligned}
$$

If the design variables are chosen such that the conditions $\Phi<0$ and $\rho>\left\|\mathbf{S}_{1} \boldsymbol{f}_{\mathrm{u}}(t)+\mathbf{S}_{2} \boldsymbol{f}_{\mathrm{m}}(t)\right\|$ are always true, then the derivative of Lyapunov candidate satisfies $\dot{V}<0$. Thus, the system will converge with the sliding plane in finite time. The signum function can induce chattering into the system. A preferred choice is to use the boundary layer method [20]

$$
\begin{aligned}
& u_{\mathrm{N}}(\sigma(t), t)=-\rho(\mathbf{S B})^{-1} \operatorname{sat}\left(\frac{\sigma(t)}{\phi}\right) \\
& \operatorname{sat}(\xi)=\left\{\begin{array}{cc}
\xi & \text { if }|\xi|<1 \\
\operatorname{sgn}(\xi) & \text { otherwise }
\end{array}\right.
\end{aligned}
$$

where $\phi$ is the boundary chosen to minimize chattering around the sliding plane. When the state trajectory moves along the sliding plane, the system 
is said to be in sliding motion, and becomes a reduced-order system with the switching function $\sigma(t)=0$. From equation (10), it is clear that the state trajectory during sliding motion is governed by

$$
\dot{\boldsymbol{e}}_{1}=\overline{\mathbf{A}}_{11} \boldsymbol{e}_{1}+\boldsymbol{f}_{\mathrm{u}}(t)
$$

The sliding surface vector is designed such that the nominal system in equation (14) gives desired performance in the face of the unmatched uncertainty.

\subsection{Fuzzy sliding surface}

Sliding mode controllers have the useful property of invariance to matched uncertainty during sliding motion. However, unmatched uncertainty continues to be a problem. In equation (14) the uncertainty term contributes to the system dynamics that govern the sliding motion. The disturbance observer is used to counteract the effects of unmatched uncertainty by estimating the ideal gripper rest position. However, the disturbance observer requires low-pass filtering and is subject to parameter model-plant mismatch. This means that the observer estimate takes time to converge, and its optimum performance is not guaranteed. It is important, therefore, that the sliding surface exhibits robustness to the unmatched uncertainty during this convergence time. This is the principle purpose of the fuzzy sliding mode: to move the sliding surface in order to achieve maximum robustness to unmatched uncertainty during convergence time of the disturbance observer. This is particularly important because the performance of the disturbance observer is sensitive to parameter model-plant mismatch, and thus the convergence time is not necessarily known or necessarily short. In this section, a sliding mode controller with a fuzzy sliding plane is proposed which uses slip and slip rate as input variables.

The sliding surface vector $\boldsymbol{S}=\left[\begin{array}{ll}\boldsymbol{S}_{1} & \boldsymbol{S}_{2}\end{array}\right]$ is redefined as a fuzzy surface vector $\tilde{\boldsymbol{S}}$. A Takegi-Sugeno fuzzylogic system is used to determine the fuzzy surface vector. The fuzzy-logic system is briefly described in this section. A more detailed description can be found in [21-23]. The fuzzy system is defined by a rule set consisting of $n$ rules, each of the form

$$
\text { Rule }[i]: \text { if } x(t) \text { is } \tilde{A}_{j} \text { and } \dot{\boldsymbol{x}}(t) \text { is } \tilde{B}_{k} \text { then } \tilde{\boldsymbol{S}} \text { is } \tilde{\boldsymbol{S}}_{l}
$$$$
\text { for } j=1,2, \ldots, c k=1,2, \ldots, c l=1,2, \ldots, c
$$

where $\tilde{A}_{j}$ and $\tilde{B}_{k}$ are fuzzy sets which span some part of the universe of discourse and $\tilde{\boldsymbol{S}}_{l}$ is the consequent or, in this case, the approximated sliding vector.
Defining $\mu_{\tilde{A}_{i}}(x(t)) \in[0,1]$ and $\mu_{\tilde{B}_{i}}(\dot{x}(t)) \in[0,1]$ as the membership functions which represent the degree of membership of $x(t)$ to $\tilde{A}_{j}$ and $\dot{x}(t)$ to $\tilde{B}_{k}$ respectively. Using the weighted average method, the fuzzy system output, i.e. the fuzzy sliding surface vector, is given by

$$
\tilde{\boldsymbol{S}}=\frac{\sum_{1}^{n} \tilde{\boldsymbol{S}}_{i} \mu_{\tilde{A}_{i}}(x(t)) \mu_{\tilde{B}_{i}}(x(t))}{\sum_{1}^{n} \mu_{\tilde{A}_{i}}(x(t)) \mu_{\tilde{B}_{i}}(x(t))}
$$

Three fuzzy sets chosen to span the universe of discourse of the slip input are $\tilde{A}_{\text {low }}, \tilde{A}_{\text {med }}$, and $\tilde{A}_{\text {high }}$, and the fuzzy sets chosen for the slip-rate input are $\tilde{B}_{\text {low }}, \tilde{B}_{\text {med }}$, and $\tilde{B}_{\text {high. }}$. The membership functions are chosen to be triangular-shaped functions. Similarly, three output sliding surface vectors are assigned to the fuzzy system output, $\tilde{\boldsymbol{S}}_{\text {low }}, \tilde{\boldsymbol{S}}_{\text {med }}$, and $\tilde{\boldsymbol{S}}_{\text {high }}$. The rule set of the fuzzy system is shown in the fuzzy associative memory (Table 1).

The tuning of FLCs, i.e. the shape of the membership functions and the choice of output vector, is a challenging problem and is the subject of ongoing research. Where $a$ priori data or training data sets are available, optimization methods, for example genetic algorithms $[\mathbf{2 4}, \mathbf{2 5}]$, can be used to empirically determine the parameters of the FLC. Alternatively, FLCs can be tuned using 'common sense' experience and trial and error. Although this approach restricts practically the allowable complexity of the fuzzy controller, it is the most simple method, and occasionally the most effective. This approach is taken in this paper. The model derived at the beginning of section 2 is used to determine the effects of different choices of the fuzzy-logic parameters. Thus, the outcome for any specific choice of variables can be assessed prior to experimental implementation.

\section{SIMULATION}

To demonstrate the efficacy of the proposed control scheme, a gripper was simulated picking up an unknown object. The system was implemented in the Simulink environment using modified equations

Table 1 Fuzzy associative memory bank

\begin{tabular}{llll}
\hline \multicolumn{3}{c}{ Slip rate } \\
\hline Slip & Low $\tilde{B}_{\text {low }}$ & Medium $\tilde{B}_{\text {med }}$ & High $\tilde{B}_{\text {high }}$ \\
\hline Low $\tilde{A}_{\text {low }}$ & $\tilde{\boldsymbol{S}}_{\text {low }}$ & $\tilde{\boldsymbol{S}}_{\text {low }}$ & $\tilde{\boldsymbol{S}}_{\text {med }}$ \\
Medium $\tilde{A}_{\text {med }}$ & $\tilde{\boldsymbol{S}}_{\text {low }}$ & $\tilde{\boldsymbol{S}}_{\text {med }}$ & $\tilde{\boldsymbol{S}}_{\text {high }}$ \\
High $\tilde{A}_{\text {high }}$ & $\tilde{\boldsymbol{S}}_{\text {med }}$ & $\tilde{\boldsymbol{S}}_{\text {high }}$ & $\tilde{\boldsymbol{S}}_{\text {high }}$ \\
\hline
\end{tabular}


(3) and (4), and equation (1) with: added backlash to define the gripper-finger motion, a $0.1 \mathrm{~ms}$ delay at the controller output. The modified equation (3) and (4), uses a non-linear continuous model for friction, to define object motion. The object was assumed to be constrained from rotation in all directions. The friction term is governed by the Lugre friction model [26]; which is a continuous friction model that is able to describe effects such as stick slip and Stribeck effect while remaining simple to implement. The Lugre parameters were chosen to be the same as those proposed in Canudas de Wit et al. [26]. All model parameters are shown in Table 2.

The control scheme was investigated by simulating the gripper catching objects with different mass, stiffness, and coefficient of friction properties. Initially, the gripper has a contact force of $0.5 \mathrm{~N}$ on the object which is held stationary. The object is then dropped. For performance comparison, a PD controller and FSMC were tuned to give less than $0.01 \mathrm{~m}$ slip for the nominal parameters in Table 2 . Both linear-feedback and FLCs have shown good results when applied to the soft-grasping problem and are adaptable to a wide range of sensor types. The general performance of a FLC, however, is difficult to gauge as it is dependent on tuning multiple degrees of freedom. Therefore, a linearfeedback controller was chosen for performance comparison with the sliding mode approach. In this case, the PD controller is the most applicable of the linear control methodologies described in the Introduction to the present problem.

The sliding mode controller was tuned using the model described in section 2, equations (1) to (5), the method described in section 2.2, and the parameters in Table 2. The tuning parameter $\rho$ was chosen to be large to guarantee system stability and reachability of the system for large uncertainty. The remaining parameters $(\Phi, \phi)$ of the sliding mode controller trial and error to give less than $0.01 \mathrm{~m}$ slip. The fuzzy logic component was chosen by varying

Table 2 Model parameters

\begin{tabular}{ll}
\hline Parameter & Value \\
\hline$M_{\text {obj }}$ & $0.3 \mathrm{~kg}$ \\
$\mu_{\mathrm{s}}$ & 0.8 \\
$\mu_{\mathrm{d}}$ & 0.6 \\
Lugre parameters $\left(\sigma_{0}, \sigma_{1}, \sigma_{2}\right.$, & $1 \times 10^{5} \mathrm{~N} / \mathrm{m}, 1 \times 10^{2} \mathrm{~N} \mathrm{~s} / \mathrm{m}, 1 \mathrm{~N} \mathrm{~s} / \mathrm{m}$ \\
$\quad$ and $\left.V_{\mathrm{s}}\right)$ & and $0.001 \mathrm{~m} / \mathrm{s}$ \\
$J$ & $0.05 \mathrm{~kg} \mathrm{~m}$ \\
$D$ & $1 \mathrm{~N} \mathrm{~s} / \mathrm{m}$ \\
$K$ & $5000 \mathrm{~N} / \mathrm{m}$ \\
$D_{\mathrm{s}}$ & $1 \mathrm{~N} \mathrm{~s} / \mathrm{m}$ \\
$n$ & $2 \times 10^{-6}$ \\
$L$ & $0.1 \mathrm{~m}$ \\
Backlash & $1.2 \times 10^{-3} \mathrm{rads}$ \\
\hline
\end{tabular}

the parameters in Table 2, and observing the sliding surface parameters required to give a similar resultant sliding motion system, that conformed to the design specification of less than $0.01 \mathrm{~m}$ slip. It was found that the position gain in the sliding vector (i.e. the component $\left(S_{12}\right)$ that multiplies by the gripper angular-position $\theta(t)$ in the switching function) changed little in response to changes in the choice of parameters. Therefore, only the slip rate gain component of the sliding vector was estimated by the FLS. Similarly, adjustments to the linear component of the control $\left(u_{\mathrm{L}}(x(t), t)\right)$ due to variation of the sliding surface are ignored as it can be classed as matched uncertainty.

The control laws for the system are shown in Table 3 and the fuzzy controller output surface in Fig. 3. Although the choice of control law parameters gives similar resultant slip, the peak contact force for the PD controller, identified by the peak gripper position is greater. The change from slip state to static state by the system is abrupt due to the discontinuity inherent in friction. Consequently, the effective damping of the PD controller after slip is minimal and overshoot results. The PD controller requires a relatively high derivative gain to match the switching of the FSMC.

\subsection{Results and discussion}

The controllers were investigated for their sensitivity to matched uncertainty $f_{\mathrm{m}}$. The gripper inertia parameter was changed in the simulation from its nominal value to $J=0.1$ to simulate model-plant mismatch. The results are shown in Fig. 4 for both the PD controller and the FSMC. The FSMC shows no significant change in performance in either the gripper position response or the resultant slip and is clearly insensitive to changes in this property. However, the PD controller shows a significant difference in performance; having an increased contact force and slip relative to the nominal system. Similar results are obtained by varying other parameters to induce matched uncertainty.

The quality of FSMC invariance was reduced when the size of the backlash and time delay were

Table 3 Control laws

\begin{tabular}{ll}
\hline Controller & Control law \\
\hline FSMC & $u_{l}=8 \dot{x}(t)-1000\left(\theta(t)-\hat{\theta}_{\mathrm{d}}(t)\right)-15 \dot{\theta}(t)$ \\
& $u_{n}=-50 \mathrm{sat}\left(\frac{s(t)}{1}\right)$ \\
& $\sigma(t)=\tilde{S}_{11} \dot{x}(t)+300\left(\theta(t)-\hat{\theta}_{\mathrm{d}}(t)\right)+\dot{\theta}(t)$ \\
PD & $u=-0.7 \dot{x}(t)-0.08 \ddot{x}(t)$ \\
\hline
\end{tabular}



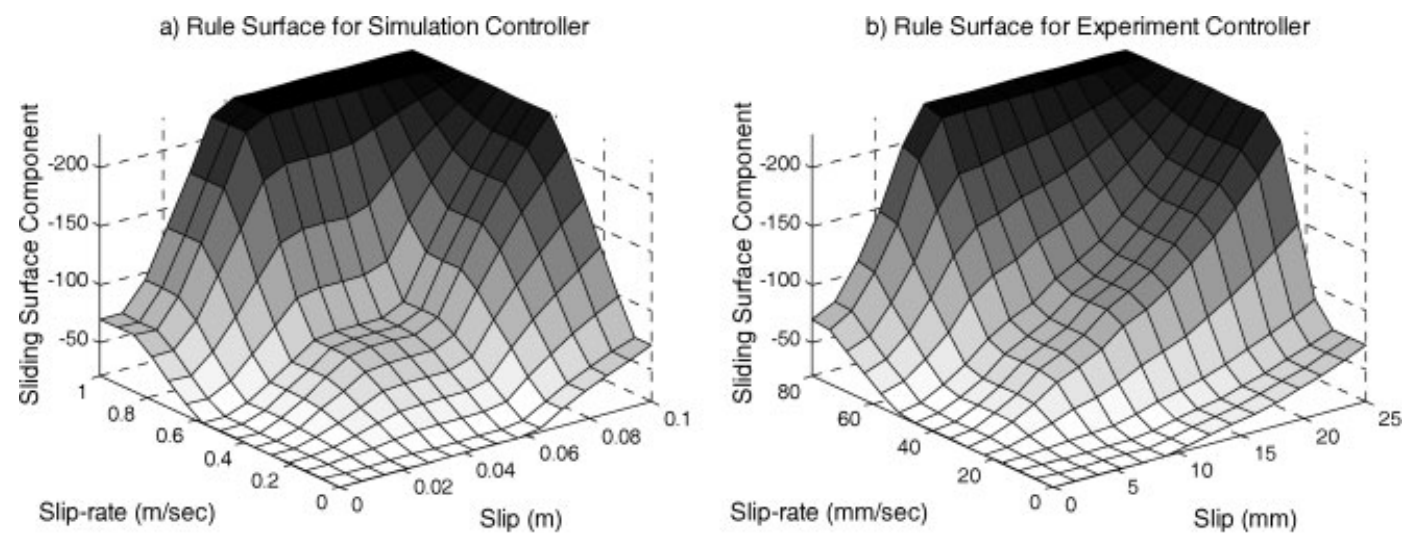

Fig. 3 Fuzzy system rule surfaces
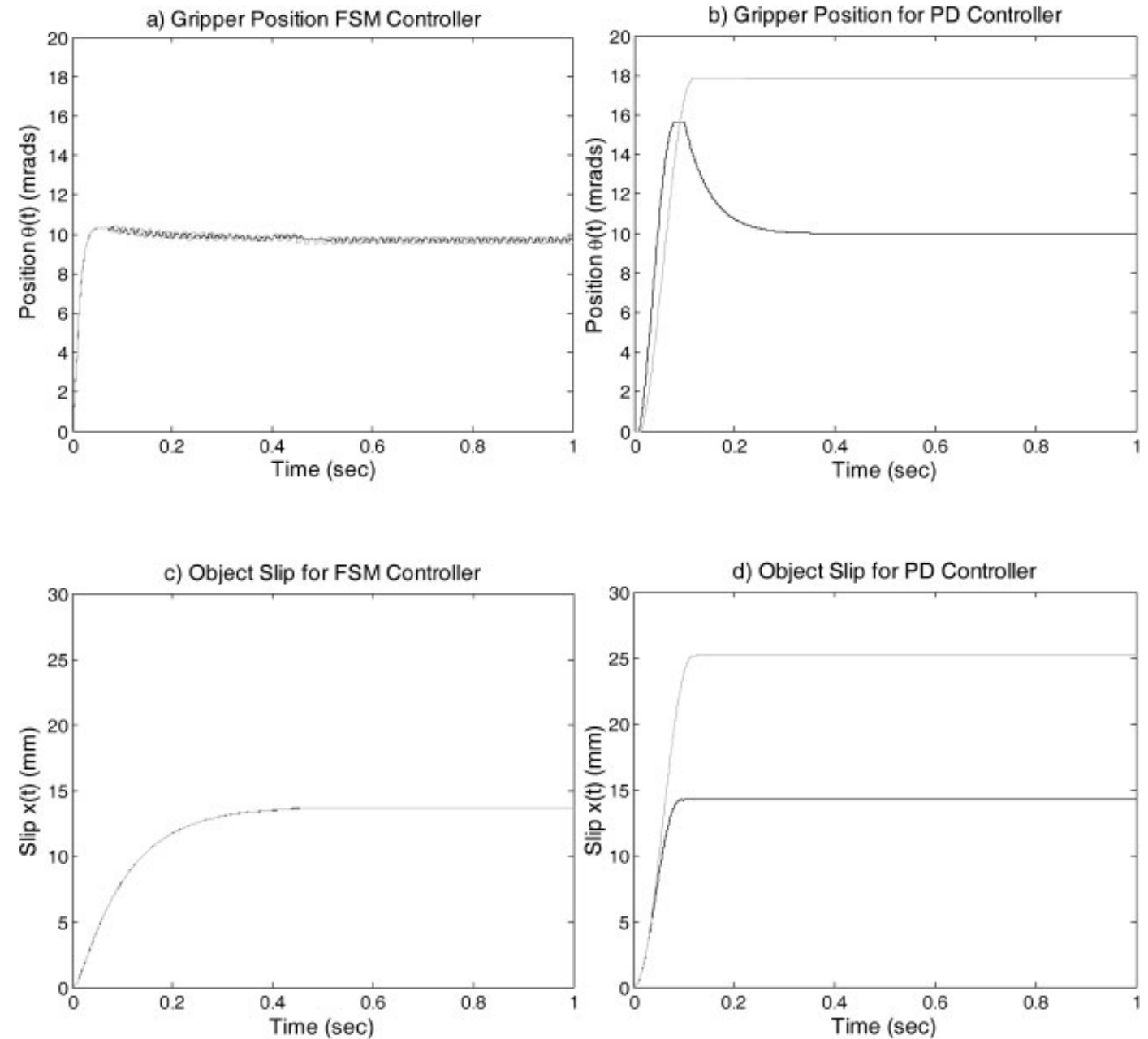

Fig. 4 Gripper position and object slip for FSMC and PD controller using $J=0.05$ (black) and $J=0.1$ (light grey)

increased - shown in Fig. 5. Although the FSMC position performance was similar, the increased chatter increased slightly the amount of slip that occurred. Chatter is extremely undesirable as it has an adverse and potentially damaging effect on the system components. The results confirms the analysis of Lee and Utkin [27], where chatter was shown analytically to be induced by unmodelled parasitic dynamics that exist in any real system. The chatter can be reduced by changing the boundary layer 

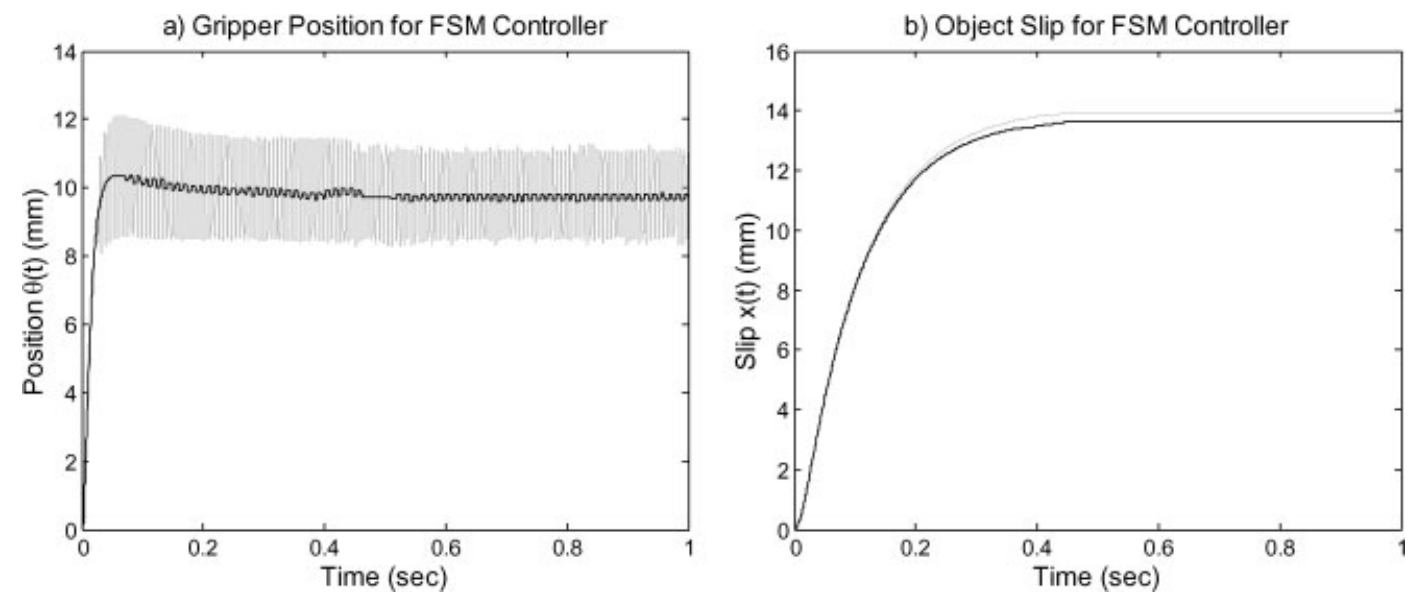

Fig. 5 Gripper position and object slip for FSMC using a $1.2 \times 10^{-3}$ rads backlash and $0.1 \mathrm{~ms}$ time-delay (black), and a $2.4 \times 10^{-3}$ rads backlash and $1 \mathrm{~ms}$ time delay (light grey)

function $\phi$ in the control (equation (13)) - shown in Fig. 6. Chattering is reduced as the controller is less sensitive to minor deviations of the state trajectories within a neighbourhood around the sliding surface. However, this insensitivity also results in steadystate error, and a loss of invariance due to the continuous control action used while the itinerant state trajectory reaches the boundary of the neighbourhood.

The object parameters were varied to induce unmatched uncertainty in the system. The controller position and slip response for the nominal parameters and the parameters of a smoother, heavier object (i.e. increased $M_{\text {obj }}$ and decreased frictional coefficients) are shown in Fig. 7. The FSMC shows an improved performance; having both a reduced peak position and lower resultant object slip. The fuzzy-logic element of the sliding function changes the sliding plane characteristics as a result of the greater slip and slip rate. Thus, in the reduced-order system of equation (14) the slip rate becomes more responsive to this state variable. The result is a decrease in slip for the heavy smooth object. In this respect, the FLS is a gain scheduling mechanism, and the performance in this test is dependent on the tuning of the input and output membership functions. Subsequent improvement in performance can be obtained by increasing slope of the fuzzy logic output surface. In addition, further improvements can be obtained by reducing the settling time of the disturbance observer. As shown in Fig. 8, the change in system parameters adversely affects the settling. The effect of slow settling time on the system response is partly alleviated by the robustness of the fuzzy sliding mode, however, it is clear that the overall performance of the control algorithm can be improved by increasing the robustness of the disturbance observer.
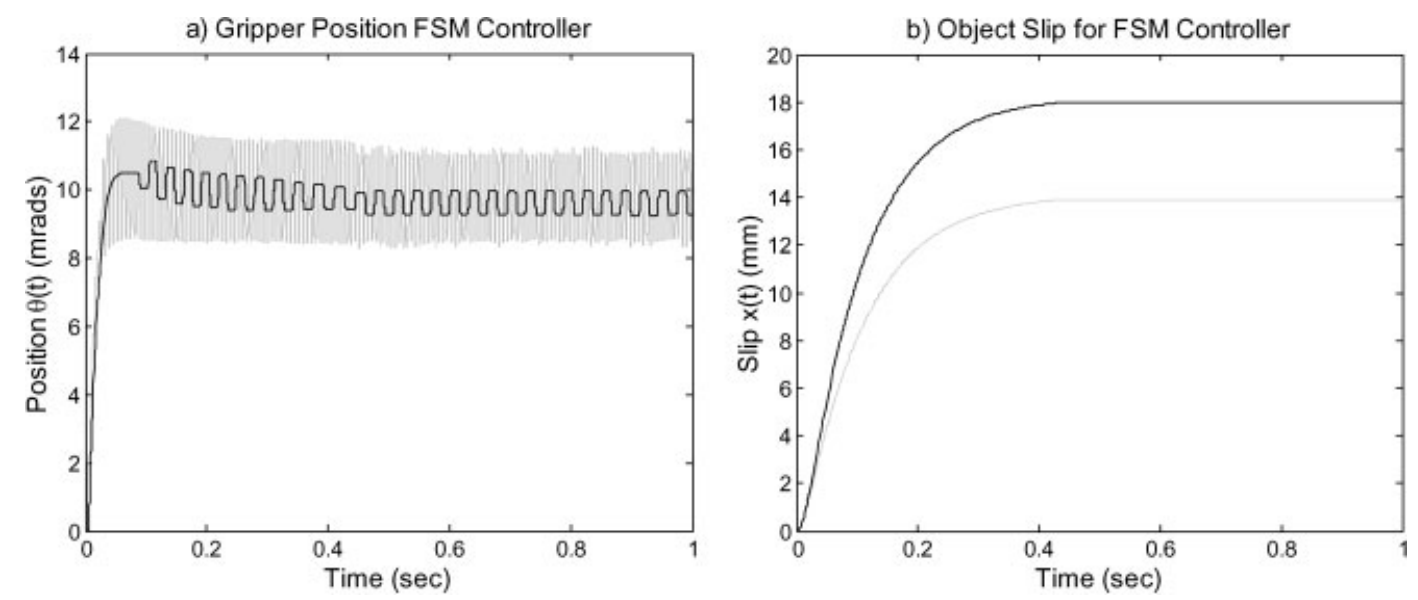

Fig. 6 Gripper position and object slip with a $2.4 \times 10^{-3}$ rads backlash and $1 \mathrm{~ms}$ time delay using $\phi=1$ (light grey) and $\phi=10$ (black) 

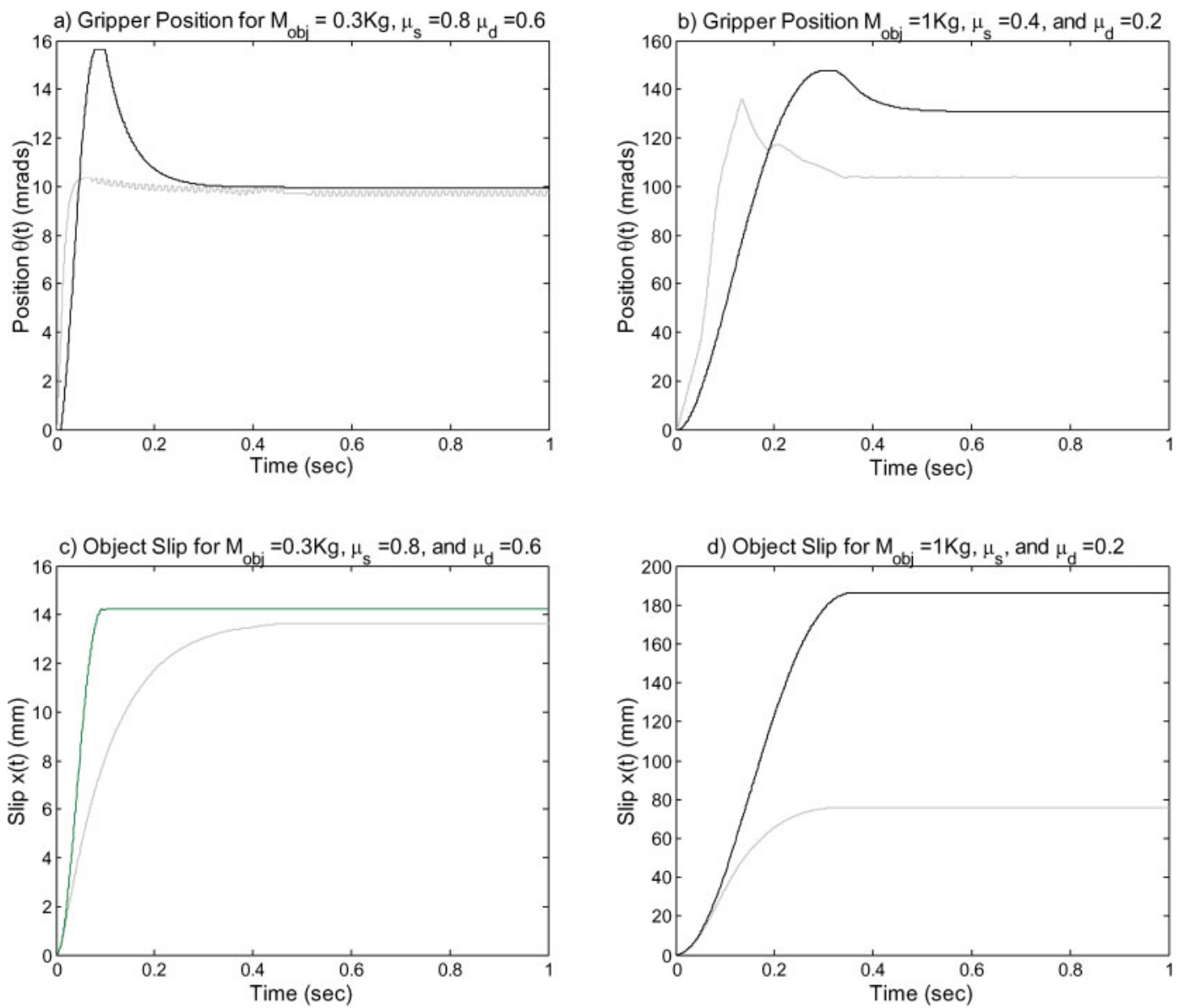

Fig. 7 Gripper position and object slip for FSM controller (light grey) and PD controller (black) using varying object properties

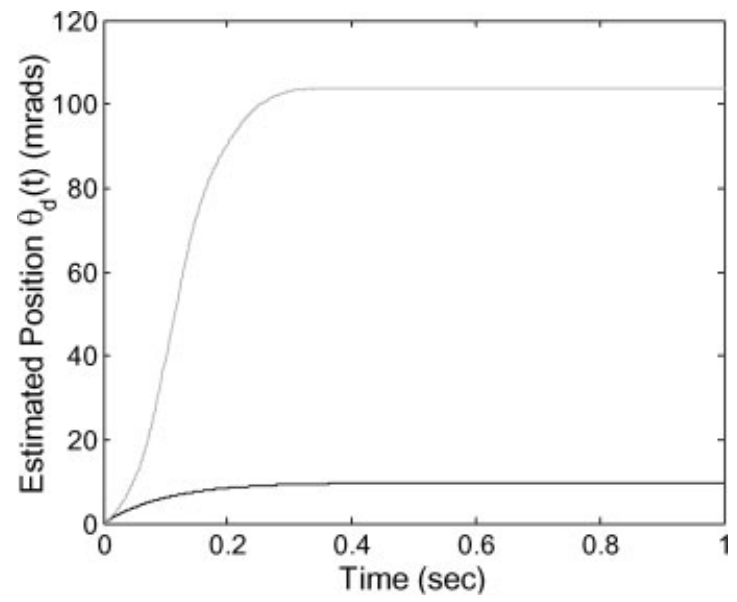

Fig. 8 Disturbance observer output for nominal object $\left(M_{\mathrm{obj}}=0.3, \quad \mu_{\mathrm{s}}=0.8, \quad \mu_{\mathrm{d}}=0.6\right) \quad$ (black), and smoother heavier object $\left(M_{\mathrm{obj}}=1 \mathrm{~kg}, \mu_{\mathrm{s}}=0.4\right.$, $\mu_{\mathrm{d}}=0.2$ ) (light grey)

\subsection{Practical implementation}

Issues related to the practical implementation of the proposed control scheme were investigated using the experimental test rig in Fig. 9. The rig was designed to assess the control algorithms' ability to reduce slip. The apparatus consists of a 3000:1 worm-geared rotary arm of $0.1 \mathrm{~m}$ length, driven by a $12 \mathrm{~V} \mathrm{DC}$ motor, and a carriage on a set of linear rails. An elastomer strip is fixed to the carriage surface. A spring is used to pre-load the arm and eliminate the system backlash. The carriage position is measured by a linear potentiometer with an output of $0.13 \mathrm{~V} / \mathrm{mm}$. The tip displacement of the rotary arm is measured by a rotary encoder which gives an approximate resolution at the tip of the arm of $3.14 \times 10^{-5} \mathrm{~mm} /$ count. The carriage is attached to a mass via a nylon cable and pulley system. The controller is implemented using a PC programmed in $\mathrm{C}++$. The output of the rotary encoder is acquired using a custom quadrature encoder reader and analogue signals are acquired using a PC30AT 12bit data acquisition card sampling at $1000 \mathrm{~Hz}$. The effective maximum quantization error is $0.04 \mathrm{~mm}$. The encoder and position sensor inputs are digitally filtered and differentiated using second-order discrete recursive filters and Euler's method respec- 

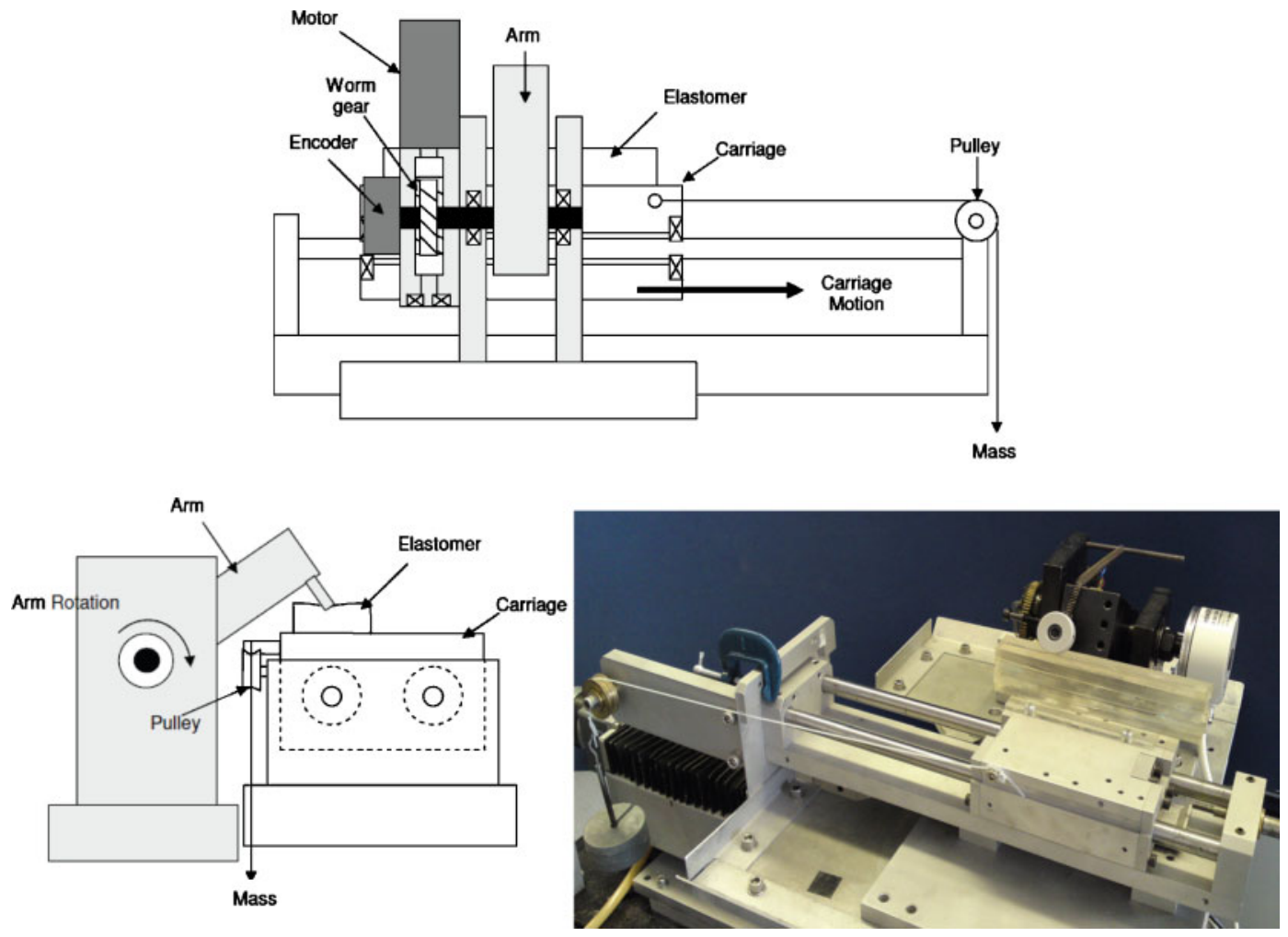

Fig. 9 Experimental test rig

tively. It is held stationary and the arm moved into contact with the elastomer layer. The carriage is then released.

The output of the fuzzy sliding mode control law in Table 3 is scaled for the experimental test rig so that torque is generated proportional to the motor voltage. The error resulting from this assumption is a form of parametric matched uncertainty and covered by the controller's invariance properties. The discontinuous injection $(\rho)$ gain is adjusted, such that the motor is just below saturation when the

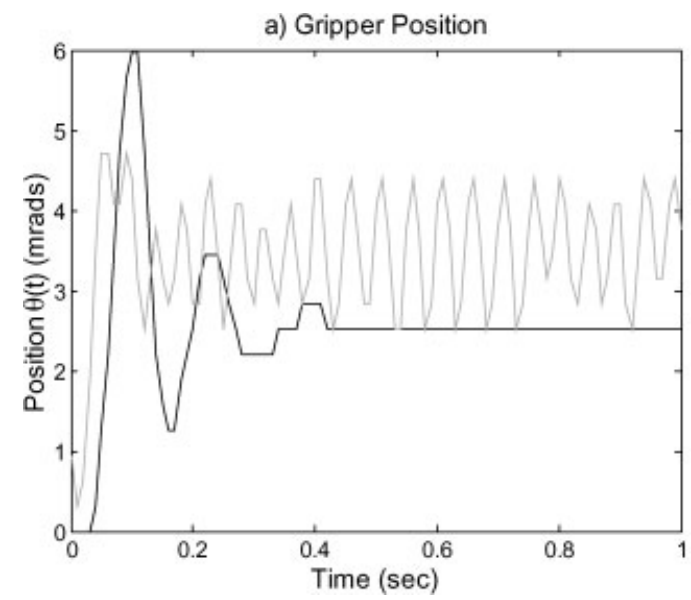

injection term $(\rho \cdot \operatorname{sat}(\sigma / \phi))$ is at maximum. Similarly, the boundary constant $\phi$ from equation (13) is adjusted to minimize chattering in the system. Finally, the fuzzy rule surface is retuned by changing the position of the membership functions on the universe of discourse for each input variable. The output variables are not retuned. The new fuzzy decision surface is shown in Fig. 3(b).

The system is typical response when the carriage is released with a $0.3 \mathrm{~kg}$ mass is shown in Fig. 10 for different boundary layer constants $\phi$. As expected,

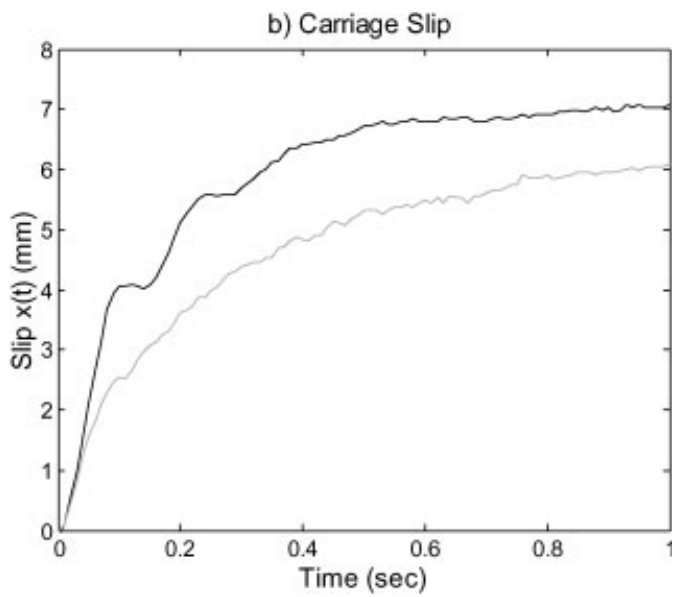

Fig. 10 Typical gripper position and carriage slip for boundary layer $\phi=1$ (black) and $\phi=0.1$ (light grey) 
the choice of a lower boundary layer constant leads to considerable chattering in the arm position, while increasing the boundary layer constant removes the chattering, but slows the response of the system. As previously discussed, while the system states are within the boundary layer, the control law is effectively a continuous controller (i.e. $u=-\rho \sigma(t) / \phi$ ). As a result, the full influence of the switching function is delayed and some invariance lost.

To assess the robustness of the control system the experimental parameters were changed significantly to induce unmatched uncertainty; the mass was increased to $0.5 \mathrm{~kg}$ and the elastomer layer was covered in a tape to produce a smooth surface. The test was repeated using $\phi=0.25$ as the boundary layer. The position and slip response are shown in Fig. 11. Chattering is evident as in the previous experiment; this leads to slipping in the system as a result oscillatory contact force.
The FLS changes the sliding vector gain, in response to high slip $(x(t))$ and slip rate $(\dot{x}(t))$, in order to make the system more sensitive to slip by reconfiguring the reduced-order system. The output gain of the fuzzy system is shown in Fig. 12(a). A negative feature is a large peak force which is induced by the transient high gain.

The controller performance is inhibited by the increased settling time of the disturbance observer as a result of changes in the experimental parameters. The position demand profiles in Fig. 12(b) show that the sensitivity of the disturbance observer performance to parametric changes, i.e. $0.3 \mathrm{~kg}$ with high friction and $0.5 \mathrm{~kg}$ with low friction, is significant to the robustness of the control scheme.

The reachability condition must be satisfied by an appropriate choice of discontinuous gain ( $\rho$ in equation (11)). However, it can be shown that increasing the gain term increases chatter in the
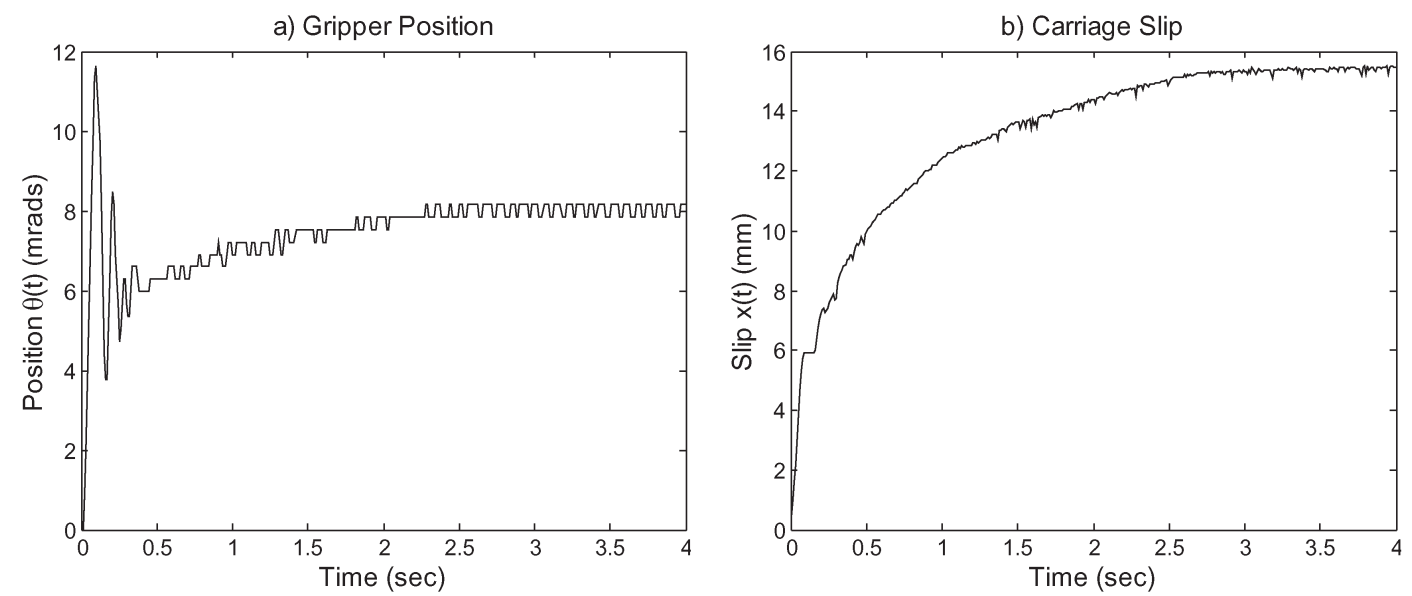

Fig. 11 Gripper position and carriage slip for low-friction surface and $0.5 \mathrm{~kg}$ mass
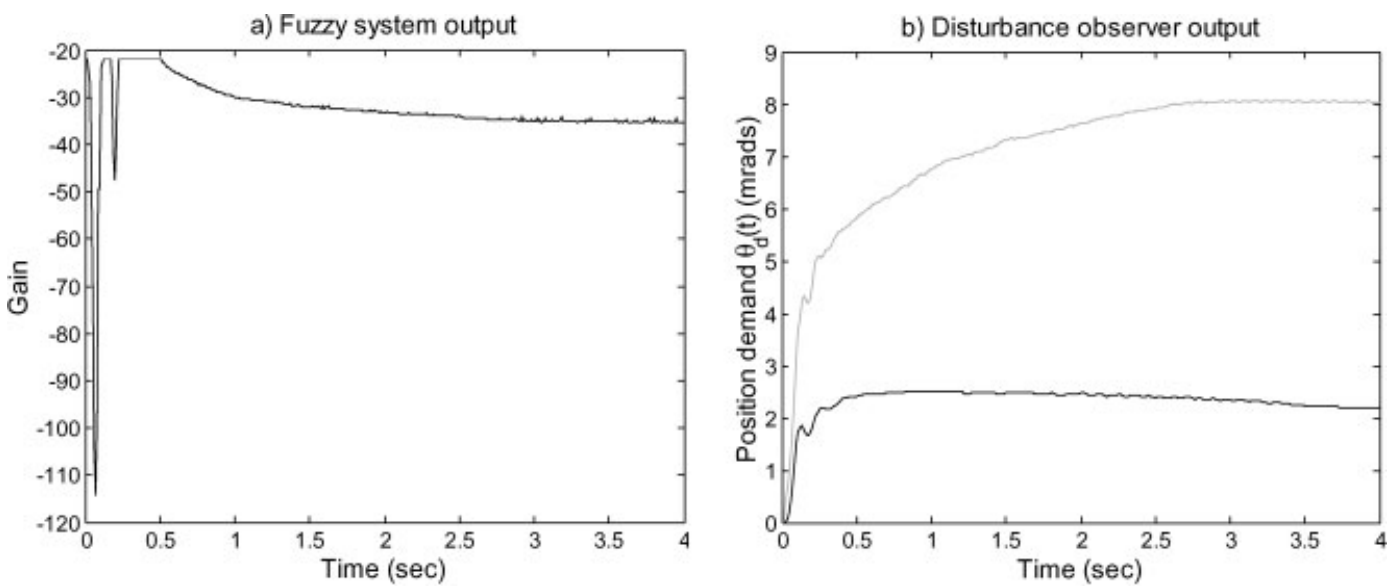

Fig. 12 (a) Fuzzy system output and (b) disturbance observer output for high-friction low-mass (black) and low-friction high-mass (light grey) cases 
system [27]. Furthermore, the actuator saturation voltage is a practical limit on the gain term. In this case, during the initial slipping phase the actuator was saturated for a short duration of time in an attempt to drive the system back to the sliding surface. Although in this case the system was still able to drive the state trajectory back to the sliding surface, it is evident that in any real system global reachability is unachievable.

\section{CONCLUSIONS}

A real-time feedback controller using a slip sensor is vital for optimal soft grasping when the object properties are uncertain. The most prevalent problem in designing such a controller is the need for a high performance response with high robustness as a result of large parameter uncertainty. In this paper, a FSMC combined with disturbance observer is proposed. The disturbance observer is used to approximate a gripper-position reference-demand signal that will stably grasp the object. The gripper-position is controlled by a sliding mode controller which can:

(a) optimally track the reference-demand signal with invariance to matched uncertainty;

(b) rapidly respond to occurrences of slip.

A FLS is used to modify the sliding plane to improve the controller response to unmatched uncertainty; the existence of which is attributed to unknown parameters of the grasped object. As a generic gripper model is used to design the controller, and the feedback variables are easily measurable (e.g. slip, slip rate, gripper position, and gripper velocity), the control scheme is applicable to a wide class of gripper configurations and thus has considerable value.

The efficacy of the control scheme was investigated both in simulations and experimentally. In the simulations, the FSMC was able to significantly outperform a conventional PD controller when subjected to matched and unmatched uncertainty induced by parametric changes to the model. Increasing the suboptimal components in the simulation, i.e. time delay and backlash, produced chattering in the gripper position response of the FSMC, which degraded the performance slightly. The effects of implementing the control scheme on a real system were investigated using an experimental test rig. The control scheme was able to demonstrate the rapid performance properties exhibited in the simulations. However, some difficulties were experienced which included; actuator saturation - pre- venting global reachability - and robustness of the disturbance observer. As in the simulations, the chattering effect and the deterioration of controller performance were noted. However, these were easily suppressed by appropriate tuning of the boundary layer algorithm. In both simulation and practice, it has been proved that the FSMC with disturbance observer scheme is a useful, high performance, and robust approach to the soft-grasping problem.

(C) Authors 2010

\section{REFERENCES}

1 Jockusch, J., Walter, J., and Ritter, H. A tactile sensor system for a three-fingered robot manipulator. In Proceedings of the IEEE International Conference on Robotics and automation, Vols 1-4, Albuquerque, New Mexico, April 1997, pp. 30803086 (IEEE Press, Piscataway, New Jersey).

2 Yamada, Y., Morita, H., and Umetani, Y. Slip phase isolating: impulsive signal generating vibrotactile sensor and its application to real-time object regrip control. Robotica, 2000, 18, 43-49.

3 Tremblay, M. R. and Cutkosky, M. R. Estimating friction using incipient slip sensing during a manipulation task. In Proceedings of the IEEE International Conference on Robotics and automation, Vols 1-3, Atlanta, May 1993, pp. 429-434 (IEEE Press, Piscataway, New Jersey).

4 Howe, R. D. and Cutkosky, M. R. Sensing skin acceleration for slip and texture-perception. In Proceedings of the IEEE International Conference on Robotics and automation, Vol 1-3, 1989, pp. 145-150 (IEEE Press, Piscataway, New Jersey).

5 Koda, Y. and Maeno, T. Grasping force control in master-slave system with partial slip sensor. In Proceedings of the IEEE/RSJ International Conference on Intelligent robots and systems, Vols 1-12, Beijing, China, October 2006, pp. 4641-4646 (IEEE Press, Piscataway, New Jersey).

6 Dominguez-Lopez, J. A., Damper, R. I., Crowder, R. M., and Harris, C. J. Adaptive neurofuzzy control of a robotic gripper with on-line machine learning. Robot. Auton. Syst., 2004, 48(2-3), 93-110.

7 Tomovic, R. and Stojiljkovic, Z. Multifunctional terminal device with adaptive grasping force. Automatica, 1975, 11(6), 567-570.

8 Kyberd, P. J., Evans, M., and Winkel, S. T. An intelligent anthropomorphic hand, with automatic grasp. Robotica, 1998, 16, 531-536.

9 Shang, Z. D., Wang, Q. Y., Han, J. H., and Xu, X. H. Sliding sensor and soft grasping of electron hydraulic servo manipulator. In Proceedings of the IEEE International Conference on Mechatronics and automation, Vols 1-3, Luoyang, China, June 2006, pp. 1459-1464 (IEEE Press, Piscataway, New Jersey). 
10 Dubey, V. N., Crowder, R. M., and Chappell, P. H. Optimal object grasp using tactile sensors and fuzzy logic. Robotica, 1999, 17, 685-693.

11 Mingrino, A., Bucci, A., Magni, R., and Dario, P. Slippage control in hand prostheses by sensing grasping forces and sliding motion. In Proceedings of the Conference on Intelligent robots and systems: advanced robotic systems and the real world, Vols 13, Munich, Germany, September 1994, pp. 18031809 (IEEE Press).

12 Birglen, L. and Gosselin, C. M. Fuzzy enhanced control of an underactuated finger using tactile and position sensors. In Proceedings of the IEEE International Conference on Robotics and automation, Vols 1-4, Barcelona, Spain, April 2005, pp. 2320-2325 (IEEE Press, Piscataway, New Jersey).

13 Engeberg, E. D. and Meek, S. G. Adaptive object slip prevention for prosthetic hands through proportional-derivative shear force feedback. In Proceedings of the IEEE/RSJ International Conference on Robots and intelligent systems, Vols 1-3, Nice, France, September 2008, pp. 1940-1945 (IEEE Press, Piscataway, New Jersey).

14 Kyberd, P. J. and Chappell, P. H. Object-slip detection during manipulation using a derived force vector. Mechatronics, 1992, 2(1), 1-13.

15 Maekawa, H., Tanie, K., and Komoriya, K. Dynamic grasping force control using tactile feedback for grasp of multifingered hand. In Proceedings of the IEEE International Conference on Robotics and automation, Vols 1-4, Minneapolis, April 1996, pp. 2462-2469 (IEEE Press, Piscataway, New Jersey).

16 Glossas, N. I. and Aspragathos, N. A. Fuzzy logic grasp control using tactile sensors. Mechatronics, 2001, 11(7), 899-920.

17 Burn, K., Short, M., and Bicker, R. Adaptive and nonlinear fuzzy force control techniques applied to robots operating in uncertain environments. $J$. Robot. Syst., 2003, 20(7), 391-400.

18 Edwards, C. and Spurgeon, S. K. Sliding mode control : theory and applications, 1998 (Taylor \& Francis, London, UK).

19 Lacraru, L. M. and Bouazza-Marouf, K. Friction compensation of an actively restrained clutch for path tracking. Proc. IMechE Part I: J. Systems and Control Engineering, 2006, 220(I5), 381-393. DOI: 10.1243/09596518JSCE186.

20 Young, K. D., Utkin, V. I., and Ozguner, U. A control engineer's guide to sliding mode control. IEEE Trans. Control Syst. Technol., 1999, 7(3), 328-342.

21 Ma, X. J., Sun, Z. Q., and He, Y. Y. Analysis and design of fuzzy controller and fuzzy observer. IEEE Trans. Fuzzy Syst., 1998, 6(1), 41-51.

22 Feng, G. A survey on analysis and design of modelbased fuzzy control systems. IEEE Trans. Fuzzy Syst., 2006, 14(5), 676-697.

23 Ross, T. J. Fuzzy logic with engineering applications, 2004 (Wiley, Chichester, UK).

24 Nguyen, V. B. and Morris, A. S. Using a genetic algorithm to fully optimise a fuzzy logic controller for a two-link-flexible robot arm. Robotica, 2009, 27, 677-687.

25 Montazeri-Gh, M. and Soleymani, M. Genetic optimization of a fuzzy active suspension system based on human sensitivity to the transmitted vibrations. Proc. IMechE, Part D: J. Automobile Engineering, 2008, 222(D10), 1769-1780. DOI: 10.1243/09544070JAUTO854.

26 Canudas de Wit, C., Olsson, H., Astrom, K. J., and Lischinsky, P. A new model for control of systems with friction. IEEE Trans. Autom. Control, 1995, 40(3), 419-425.

27 Lee, H. and Utkin, V. I. Chattering suppression methods in sliding mode control systems. Ann. Rev. Control, 2007, 31(2), 179-188.

\section{APPENDIX}

\section{Notation}

\begin{tabular}{|c|c|}
\hline A, B & system matrices \\
\hline$D_{\mathrm{e}}$ & effective damping of the mechanism \\
\hline$D_{\mathrm{s}}$ & viscous friction coefficient \\
\hline$\underset{\sim}{\boldsymbol{e}}(t)$ & error state vector \\
\hline$\tilde{f}(t), \tilde{\Psi}(t)$ & uncertainty bounds \\
\hline $\begin{array}{c}f_{\mathrm{m}}(\tau, t), \\
\Psi(\dot{x}(t), \\
\theta(t), t), \\
f_{\mathrm{u}}(t)\end{array}$ & uncertainty terms \\
\hline$J_{\mathrm{e}}$ & effective inertia of the mechanism \\
\hline$K$ & $\begin{array}{l}\text { combined object and fingertip } \\
\text { stiffness }\end{array}$ \\
\hline$L$ & $\begin{array}{l}\text { length from gripper axis to point of } \\
\text { contact }\end{array}$ \\
\hline$M_{\text {obj }}$ & grasped object mass \\
\hline$n$ & load transmission constant \\
\hline$S$ & sliding surface parameter vector \\
\hline$\tilde{\boldsymbol{S}}$ & fuzzy sliding vector \\
\hline $\begin{array}{r}\left.u_{\mathrm{L}}(x(t), t)\right) \\
\quad u_{\mathrm{N}}(s(t), t)\end{array}$ & control laws \\
\hline$\dot{x}(t)$ & object slip rate \\
\hline$\ddot{x}_{\mathrm{g}}(t)$ & gripper acceleration \\
\hline
\end{tabular}

$\alpha$

cosine angle between the direction of the slip of the object relative to the vertical

$\theta(t)$
$\hat{\theta}_{\mathrm{d}}$
$\theta_{0}$
$\quad \mu_{\tilde{A}_{i}}(x(t))$,
$\quad \mu_{\tilde{B}_{i}}(\dot{x}(t))$
$\mu_{\mathrm{s}}$
$\sigma(t)$
$\tau(t)$
gripper-finger angle from fully open disturbance observer output gripper-finger angle at initial contact with the object membership functions coefficient of friction switching function motor torque 